\title{
Estrutura Populacional do Camarão Sete-Barbas Xiphopenaeus kroyeri (Heller, 1862), na Foz do Rio Itajaí- Açú, Itajaí, SC, Brasil.
}

\author{
Joaquim Olinto Branco ${ }^{* 1,2}$, Maria José Lunardon-Branco², Flávio Xavier Souto ${ }^{1}$, Cláudio \\ Rogério Guerra 1 . \\ 1 - Faculdade de Ciências do Mar - FACIMAR / UNIVALI. Cx. Postal 360, 88301-970, Itajaí, SC. 2 - UFSCar. \\ P.P.G/E.R.N. CX. P. 676, 13565-905 São Carlos-SP.
}

\begin{abstract}
Xiphopenaeus kroyeri is the most exploited shrimp specie along Center-South coast of Santa Catarina State, Brazil, by artisanal fishery. In the period from March/1996 to February/1997, 3965 shrimps were collected (1843 males and 2122 females) sampled by over-trawl, with the depth oscillating between 5 and 15 meters. Xiphopenaeus kroyeri shows seasonal fluctuations in its abundance with higher rates in autumn. The population's average total-length varied between $7.5 \mathrm{~cm}$ and $9.1 \mathrm{~cm}$, and the presence of juveniles was common along the year. The males and females get to the first gonadal maturation with $7.4 \mathrm{~cm}$ and $9.0 \mathrm{~cm}$ length, respectively; these lengths were reached within 6 and 8 months of age.
\end{abstract}

Key Words: Xiphopenaeus kroyeri, Population structure, sea-bob-shrimp.

\section{INTRODUÇÃO}

O camarão sete-barbas Xiphopenaeus kroyeri (Heller, 1862), apresenta uma ampla distribuição geográfica no Atlântico Ocidental, ocorrendo da Carolina do Norte (USA) ao Estado de Santa Catarina (BR) (Holthuis, 1980). Habita águas costeiras rasas com fundo de areia e lama até 30m de profundidade (Iwai, 1973).

A espécie não apresenta estratificação populacional, sendo comum a ocorrência de juvenis e adultos na mesma área (Vieira, 1947; Iwai, 1973). Ao contrário dos camarões do gênero Penaeus, X. kroyeri não depende dos estuários para o desenvolvimento dos juvenis (Neiva \& Wise, 1963). Entretanto, a presença em zonas estuarinas está ligada a penetração de cunha salina (Holthuis, 1980). Este estudo teve como objetivo caracterizar a estrutura populacional de X. kroyeri na Foz do Rio ItajaíAçú, SC.

\section{MATERIAL E MÉTODOS}

As coletas foram realizadas mensalmente, durante o período de março/96 a fevereiro/97, em três pontos, tradicionalmente utilizados pela pesca artesanal, na Foz do Rio Itajaí-Açú, entre as Praias de Navegantes e Atalaia (26 $20^{\circ}$ ' $26^{\circ} 23^{\prime} \mathrm{S}$; 48 $36^{\prime}-48^{\circ} 33^{\prime}$ W) (Fig. 1). A profundidade nas áreas de arrasto variou entre 5 a 15 metros. Nas coletas foram utilizadas duas redes-de arrasto com portas, com 6,0 metros de abertura de boca, malha de $3,0 \mathrm{~cm}$ na manga e no corpo e $2,0 \mathrm{~cm}$ no ensacador. O tempo de cada arrasto foi de 30 minutos com velocidade média da baleeira de 2 nós.

\footnotetext{
Autor para correspondência
} 


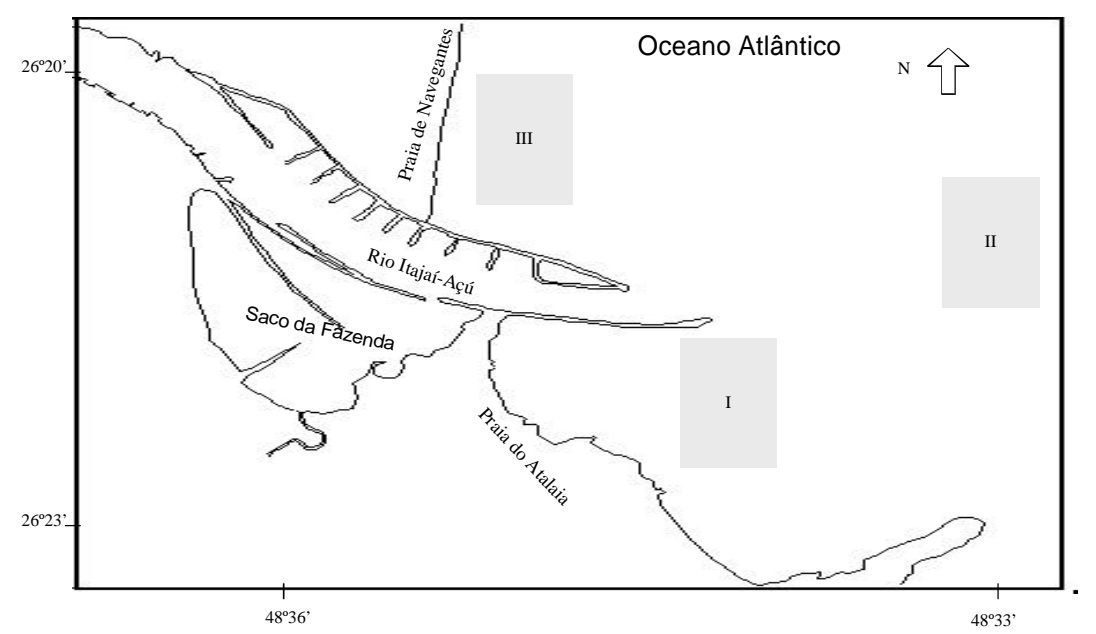

Fig. 1. Mapa indicando as áreas de coleta.

Paralelamente às coletas de camarão, foram registrados dados de temperatura $\left({ }^{\circ} \mathrm{C}\right)$ e salinidade ( ) da água de fundo, sendo que as amostras de água foram obtidas com auxílio de garrafas de Van Dorn. Imediatamente à coleta de água foi determinada a temperatura com auxílio de termômetro e a salinidade com refractômetro.

Para cada exemplar de X. kroyeri foi registrado o sexo, comprimento total $(\mathrm{Lt})$ e da carapaça (Lc) em cm e o peso total (Wt) em gramas. O estádio de maturação dos machos foi determinado pela união dos lóbulos do petasma conforme Pérez Farfante (1970); nas fêmeas pela coloração e desenvolvimento das gônadas (Vieira, 1947).

Para verificar a possível diferença entre os sexos durante os meses e por classe de comprimento, foi utilizado o teste do $\mathrm{x}^{2}$ com nível de significância de 5\% e n-1 graus de liberdade.

A relação comprimento total/comprimento do cefalotórax foi obtida graficamente para machos e fêmeas, através da dispersão dos pontos empíricos e analiticamente pela expressão: $\mathrm{Lc}$ = B.Lt, onde: $\mathrm{B}$ é constante da relação linear. A relação peso/comprimento total foi calculada para cada sexo, de acordo com Santos (1978).
O tamanho de primeira maturação ( $\left.\mathrm{Lt}_{\mathrm{PM}}\right)$, que corresponde à frequência de $50 \%$ de indivíduos adultos, foi ajustado segundo Vazzoler (1981).

As curvas de crescimento em comprimento total foram estimadas para machos e fêmeas pelo método da distribuição de frequência. A validade da expressão de Bertalanffy (1938) foi testada previamente, com a aplicação da transformação de Walford (1946) e os parâmetros estimados conforme santos (1978). As curvas de crescimento em peso foram obtidas pelo método dedutivo (Santos, 1978), após o conhecimento da relação peso/comprimento total para machos e fêmeas. A ANOVA $(p<0,05)$ foi utilizada para verificar a influência da temperatura e salinidade da água de fundo na abundância do camarão sete-barbas.

\section{RESULTADOS}

A temperatura da água de fundo nas três áreas de coleta, apresentou uma flutuação sazonal com valores mais elevados nos meses de primavera e verão, e baixos no final do outono e inverno (Fig. 2a). Os maiores valores foram registrados entre novembro, dezembro a março com temperaturas variando entre 26,0 a $27,0^{\circ} \mathrm{C}$, e as menores, em julho e agosto entre 17,0 a $19,0^{\circ} \mathrm{C}$ (Fig. 2a). O padrão de variação da temperatura manteve-se 
uniforme entre as áreas de amostragens, ocorrendo oscilações entre as médias das estações: primavera $\left(24,8^{\circ} \mathrm{C}\right)$, verão $\left(26,0^{\circ} \mathrm{C}\right)$, outono $\left(22,1^{\circ} \mathrm{C}\right)$ e inverno $\left(18,8^{\circ} \mathrm{C}\right)$.

A salinidade apresentou uma flutuação sazonal (Fig. 2b) com valores médios mais elevados na primavera $(32,7$ ) e outono $(32,0)$, e os menores no verão $(25,6$ ) e inverno $(29,1$ ). Os maiores teores de salinidade $(35,0 \quad)$ foram registrados em maio, outubro e novembro, e os menores (20,0 ) em janeiro (Fig. 2b).

Para o total de $X$. kroyeri capturados, as frequências relativas das fêmeas $(53,5 \%)$ e dos machos $(46,5 \%)$ foram diferentes do esperado de 1:1 na população (Fig. 3). Esta tendência foi confirmada, analisando-se os meses do ano através do $x^{2}$, que indicou diferenças significativas $(\mathrm{p}<0,05)$ a favor das fêmeas nos meses de maio, julho, agosto, setembro e fevereiro, e dos machos, apenas em outubro (Fig. 3).

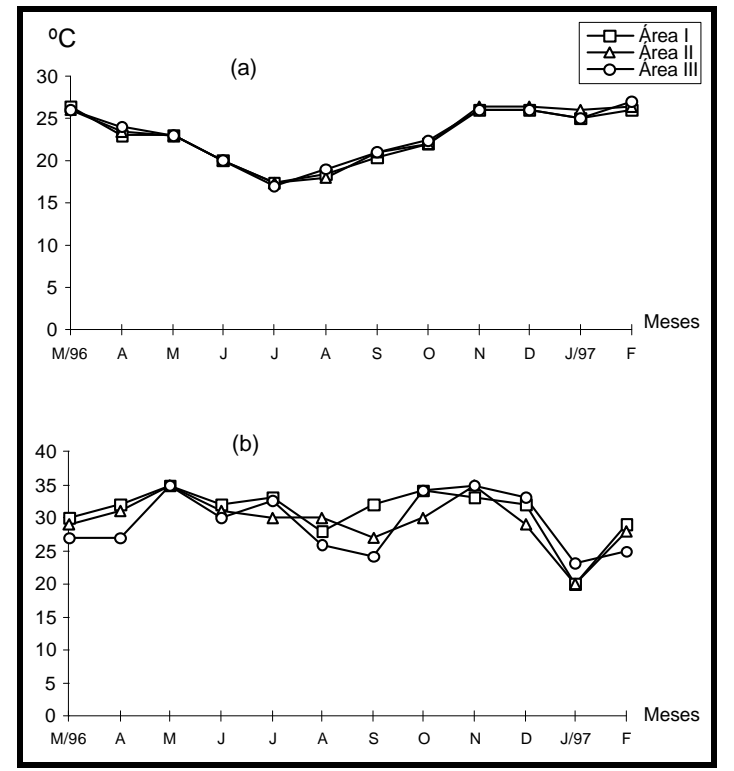

Fig. 2. Variações mensais da temperatura (a) e salinidade (b) da água de fundo.

Nos demais meses, foi observado um equilíbrio na proporção sexual da população.
As distribuições de frequências relativas de machos e fêmeas por classe de comprimento total, apresentaram uma amplitude de comprimento entre 4,0 a $12,0 \mathrm{~cm}$ (machos) e 4,0 a $14,0 \mathrm{~cm}$ (fêmeas) (Fig. 4).

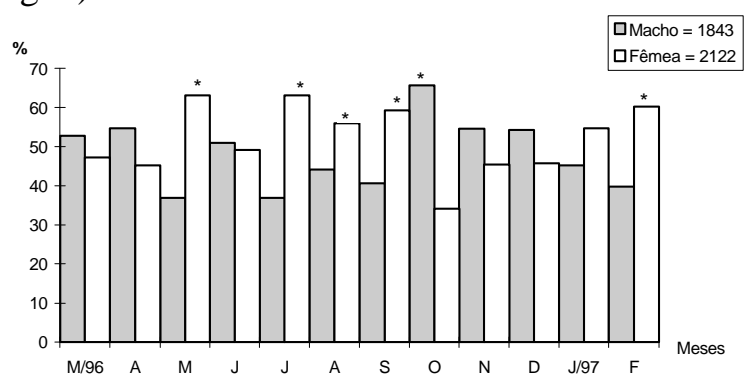

Fig. 3. Xiphopenaeus kroyeri. Frequência de ocorrência (\%) por sexo, durante o período de março/96 a fevereiro/97. * - indica diferença significativa $\mathrm{x}^{2}(\mathrm{p}<$ $0,05)$.

Foram observadas diferenças significativas a favor das fêmeas entre as classes de 4,0 a 6,0cm e 11,0 a $12,0 \mathrm{~cm}$, enquanto que nas classes de maior ocorrência, houve um equilíbrio entre os sexos (Fig. 4).

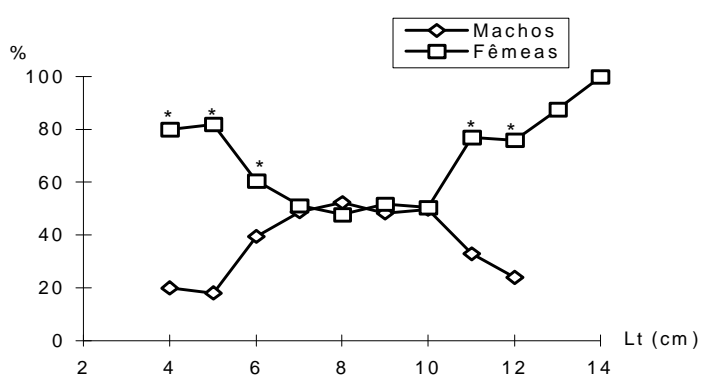

Fig. 4. Xiphopenaeus kroyeri. Frequência de Ocorrência (\%) por classe de comprimento total de machos e fêmeas. * - indica diferença significativa $\mathrm{x}^{2}(\mathrm{p}<0,05)$

A análise de Figura 5 indica que a distribuição espaço-temporal da abundância relativa de $X$. kroyeri apresenta flutuações sazonais, com os maiores índices de captura ocorrendo durante o outono, declinando gradativamente, até atingir as menores CPUE na primavera, independente da área de amostragem (Fig. 5). A ANOVA ( $F=31,8$; Gl: 621) indicou que a abundância relativa do camarão sete-barbas na Foz do Rio Itajaí-Açú está significativamente relacionada com as oscilações da temperatura e salinidade da água de fundo ao longo do ano. 
A estrutura sazonal de comprimentos da população (Fig. 6) apresentou componentes bimodais, unimodal na maioria dos casos, com o comprimento total médio oscilando entre 7,5 a $9,1 \mathrm{~cm}$. A ocorrência de juvenis na área de estudo parece se dar ao longo do ano, podendo ser representado pela captura de exemplares entre 4,0 a $7,0 \mathrm{~cm}$, principalmente no outono e inverno.

$\mathrm{Na}$ primavera, foram observados os maiores comprimentos médios da população de $X$. kroyeri na Foz do Rio Itajaí-Açú; embora com as menores frequências de ocorrência do ano (Fig. 6). No verão, ocorreu um incremento significativo na captura, mantendo $\mathrm{o}$ comprimento médio em torno dos $8,3 \mathrm{~cm}$. Durante o outono, foram registradas as maiores capturas do período de coleta, e os menores comprimentos médios da população entre $7,5 \mathrm{e}$ $7,6 \mathrm{~cm}$.

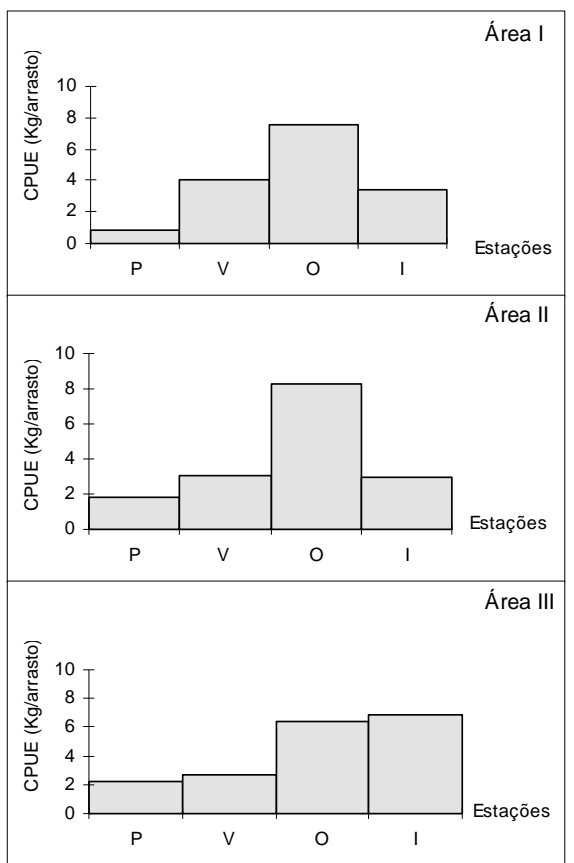

Fig. 5. Xiphopenaeus kroyeri. Distribuição sazonal das CPUE (Kg/arrasto), por área de coleta.

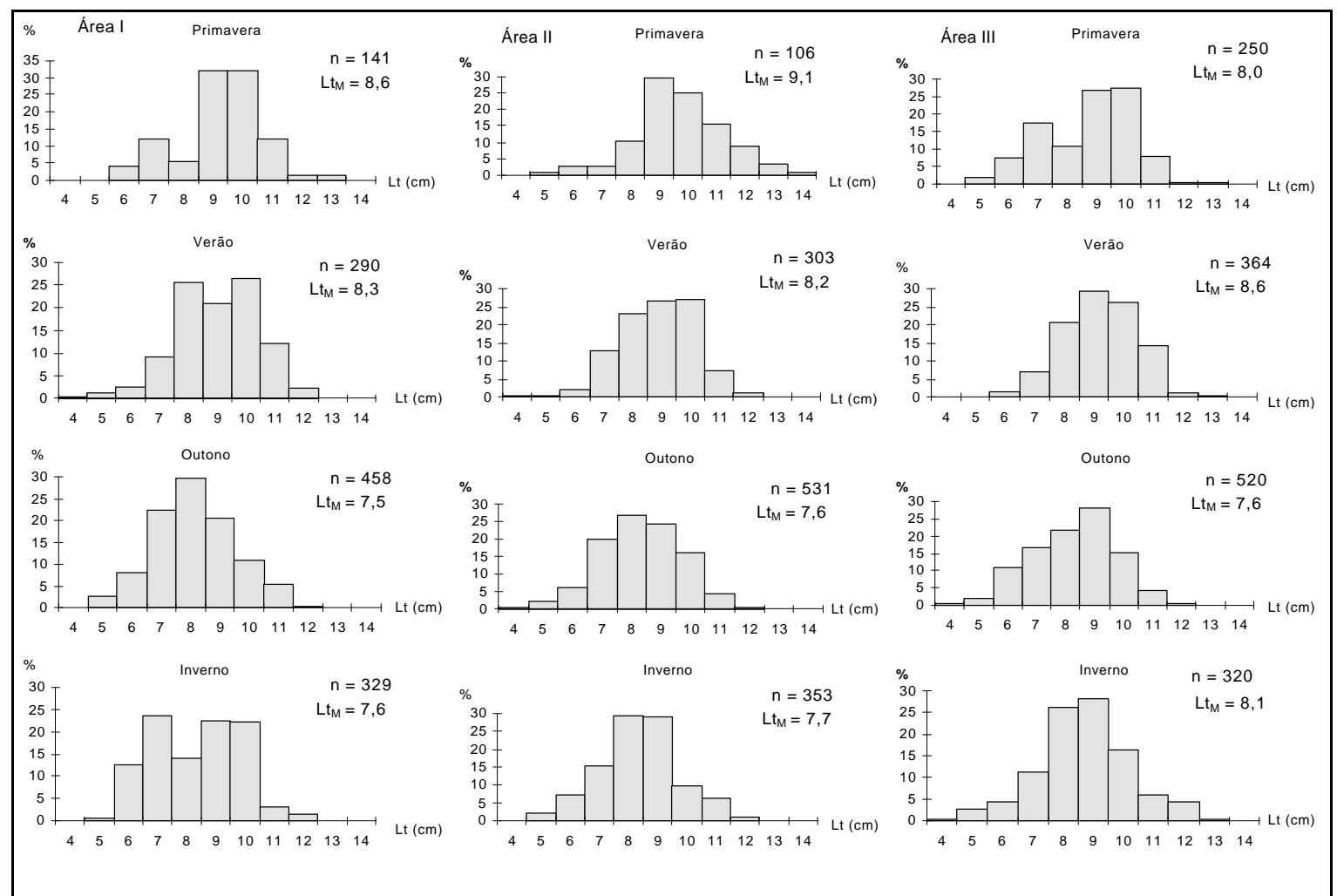

Fig. 6. Xiphopenaeus kroyeri. Distribuição de frequência das classes de comprimento total, por área de coleta e estação do ano. $\mathrm{Lt}_{\mathrm{M}}=$ comprimento total médio. 
No inverno, houve queda abrupta na frequência relativa de camarões nas áreas de coleta, ocasionando oscilações no comprimento médio, que manteve-se entre 7,6 a 8,1 cm (Fig. 6).

O tamanho de primeira maturação gonadal foi estimado em $7,4 \mathrm{~cm}$ para os machos e $9,0 \mathrm{~cm}$ para as fêmeas; a partir do comprimento total de $11,0 \mathrm{~cm}$, todos os camarões coletados eram adultos (Fig. 7). A distribuição espacial de $X$. kroyeri por comprimento total apresenta um padrão unimodal com predomínio das classes de 7,0 a 10,0cm (Fig. 8). A maior frequência de ocorrência foi registrado na Área III, seguido das Áreas II e I.

Analisando-se a população do camarão sete-barbas em função do tamanho de primeira maturação, verificou-se que a pesca artesanal praticada na Foz do Rio Itajaí-Açú, está atuando com maior intensidade sobre os juvenis (fêmeas $=70,4 \%$, machos $=46,1 \%)$ em relação ao estoque de adultos (fêmeas $=29,6 \%$, machos $=53,9 \%$ ) (Fig. 8).

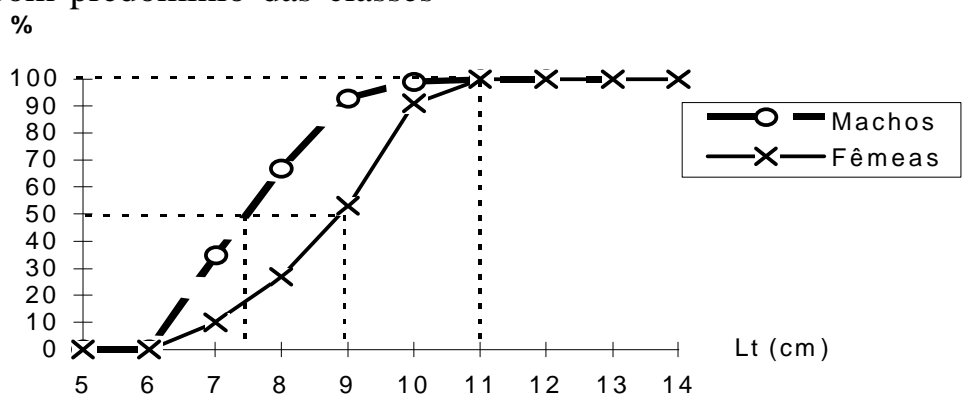

Fig. 7. Xiphopenaeus kroyeri. Distribuição de frequência de machos e fêmeas adultas, por classe de comprimento total. Machos $-\mathrm{Lt}_{\mathrm{PM}}=7,4 \mathrm{~cm}$; Fêmeas $-\mathrm{Lt}_{\mathrm{PM}}=9,0 \mathrm{~cm}$.

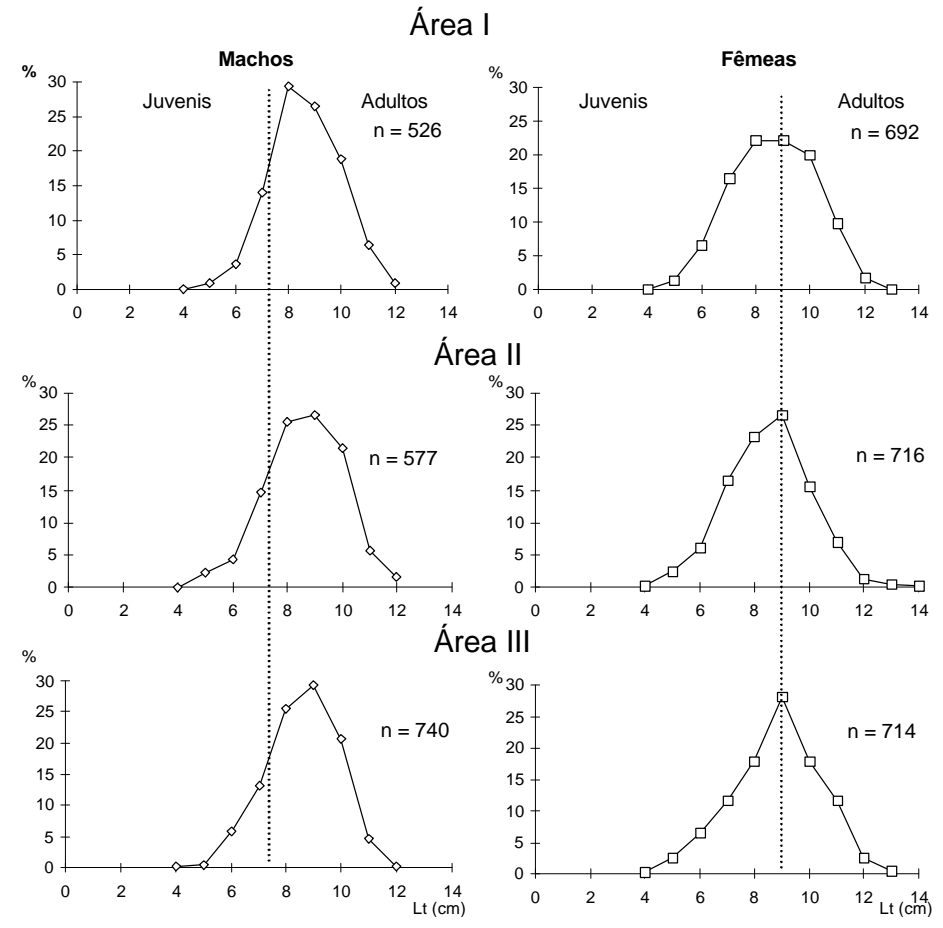

Fig. 8. Xiphopenaeus kroyeri. Distribuição de frequência das classes de comprimento total por estação de coleta e sexo. 
Os valores do peso total foram lançados em gráfico em função do comprimento total, os quais foram corroborados pela linearidade das relações (Fig. 9a, 9b) e pelos valores do coeficiente de determinação.
A relação comprimento da carapaça /comprimento total foi determinada para machos (Fig. 10a) e fêmeas (10b).
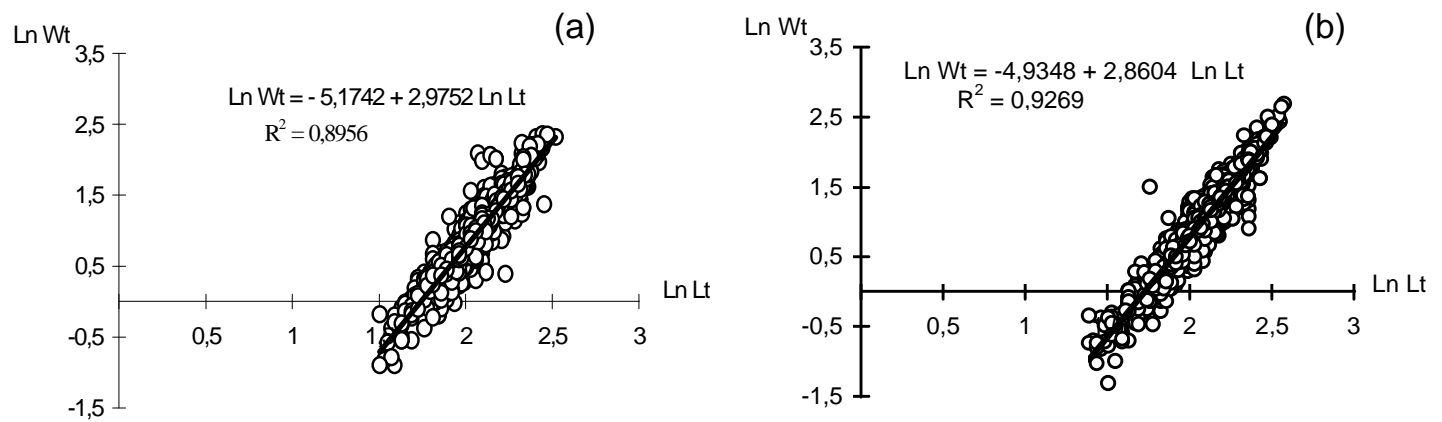

Fig. 9. Xiphopenaeus kroyeri. Transformação logarítmica da relação peso/comprimento total para machos (a) e fêmeas (b).

Considerando que ocorre dimorfismo sexual, quanto ao tamanho e a existência de relação linear pela origem. Assim, as expressões representam o crescimento relativo entre $\mathrm{Lc}$ e $\mathrm{Lt}$ e a constante da regressão, corresponde ao índice biométrico, demonstrado nas expressões:

Machos $\mathrm{Lc}=0,1879 \mathrm{Lt}, \mathrm{r}=0,9398$

Fêmeas $\mathrm{Lc}=0,1911 \mathrm{Lt}, \mathrm{r}=0,9487$.
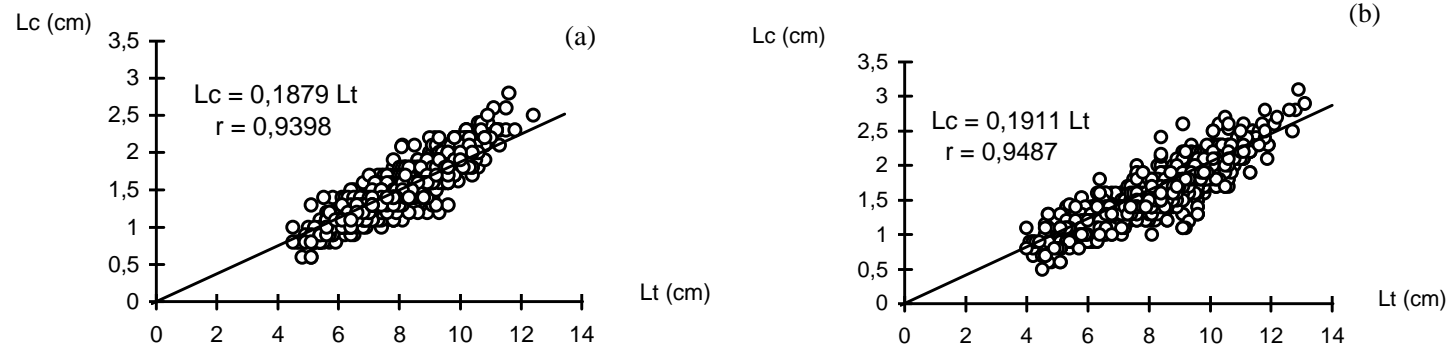

Fig. 10. Xiphopenaeus kroyeri. Relação comprimento total/comprimento cefalotórax para machos (a) e fêmeas (b).

$\mathrm{O}$ índice biométrico permite estimar o valor de uma variável através do conhecimento da outra. Para determinado comprimento total, corresponderá um comprimento de carapaça, geralmente maior nas fêmeas que nos machos.

As curvas de crescimento em comprimento total são descritas pelas equações:

$$
\begin{aligned}
& \text { Machos } \mathrm{Lt}=12,2\left(1-\mathrm{e}^{-0,2352 \mathrm{t}}\right) \quad \text { Fêmeas } \mathrm{Lt}= \\
& 14,1\left(1-\mathrm{e}^{-0,2796 \mathrm{t}}\right)
\end{aligned}
$$

Nas Figuras 11a, 11b, encontram-se as curvas teóricas de crescimento em comprimento ajustadas aos pontos empíricos para machos e fêmeas. 

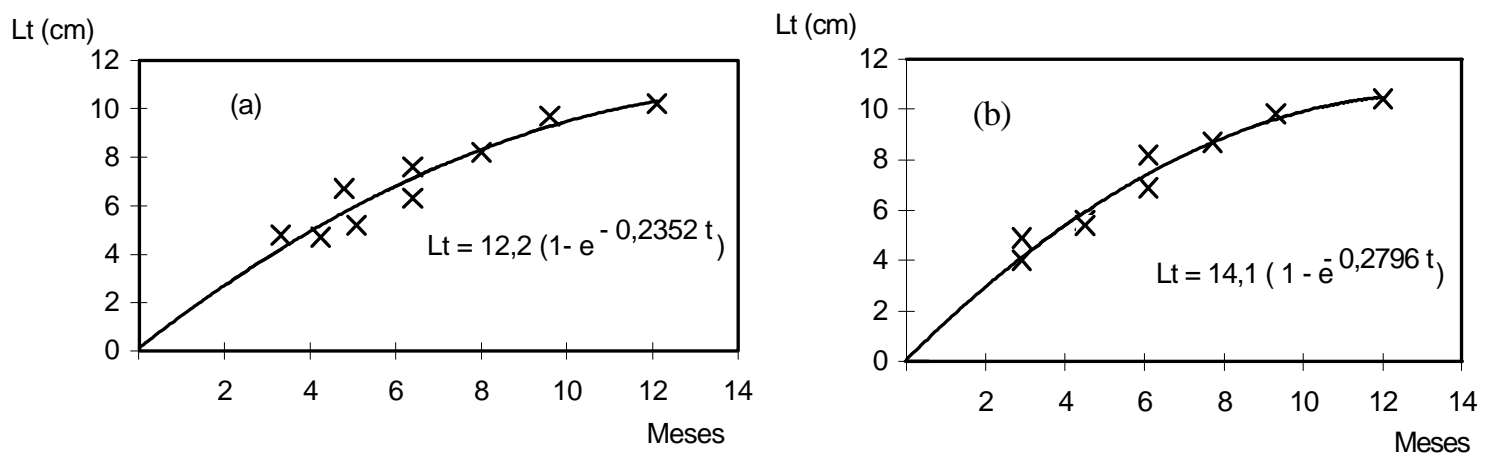

Fig. 11. Xiphopenaeus kroyeri. Curva de crescimento em comprimento total para machos (a) e fêmeas (b).

O crescimento em comprimento da carapaça (cm) foi estimado a partir das relações entre os índices biométricos e os valores de $\mathrm{L}_{\infty}$ das equações de crescimento em comprimento total, resultando nas seguintes expressões:

Machos Lc $=2,29\left(1-\mathrm{e}^{-0,2352 \mathrm{t}}\right)$ Fêmeas $\mathrm{Lc}=$ $2,70\left(1-\mathrm{e}^{-0,2796 t}\right)$.

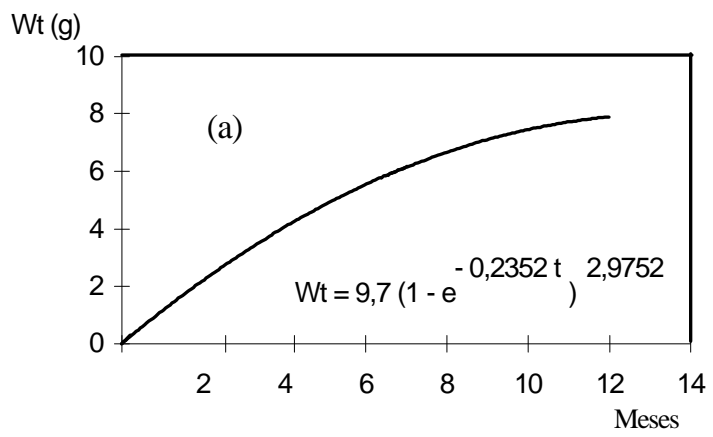

$\mathrm{O}$ crescimento em peso de $X$. kroyeri encontra-se representado nas Figuras 12a, 12b, cujas curvas apresentam as seguintes expressões:

Machos Wt $=9,7\left(1-\mathrm{e}^{-0,2352 \mathrm{t}}\right)^{2,9752}$ Fêmeas Wt $=$ $13,9\left(1-\mathrm{e}^{-0,2796 t}\right)^{2,8604}$

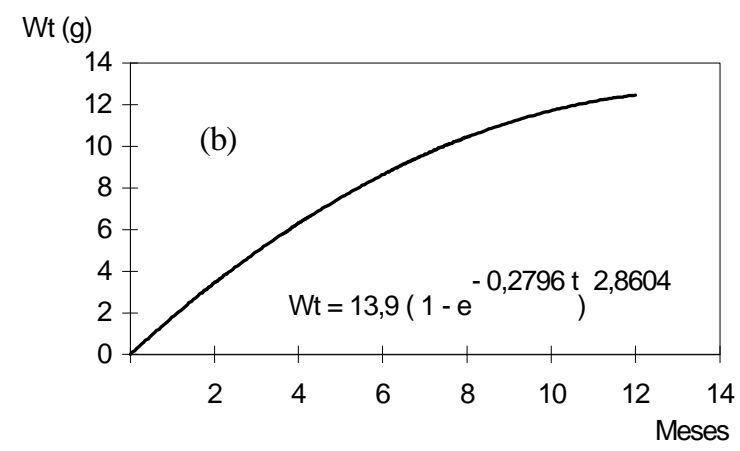

Fig. 12. Xiphopenaeus kroyeri. Curva de crescimento em peso para machos (a) e fêmeas (b).

\section{DISCUSSÃO}

O padrão sazonal da temperatura da água de fundo na Foz do Rio Itajaí-Açú, está de acordo com o esperado para a região (Matsuura, 1986); enquanto que as flutuações nos teores de salinidade podem ser associados à contribuição fluvial do Rio Itajaí-Açú.

Embora a temperatura da água tenha sido apontada como o principal fator que afeta a abundância, o crescimento e a sobrevivência de juvenis do gênero Penaeus em áreas de criadouros (D'Incao, 1984; Staples \& Haeles,
1991; Villela et al., 1997), a salinidade pode alterar os efeitos da temperatura em muitos processos metabólicos que interferem no comportamento e crescimento desses animais (Gunter, 1950; Kinne, 1964).

Nesse estudo, as diferenças de temperaturas entre verão/inverno, na área de estudo, foram em média de $7,2^{\circ} \mathrm{C}$. Já os extremos de salinidade, ficaram em torno de $15,0 \%$. Assim, a diferença significativa entre a abundância de $X$. kroyeri e a salinidade (ANOVA, $\quad \mathrm{p}<0,05$ ), provavelmente está relacionada à descarga fluvial do Rio Itajaí-Açú. No caso da temperatura, o padrão sazonal característico na abundância da espécie associado as oscilações 
da temperatura entre os meses de amostragens pode ter contribuído significativamente na diferença observada. Dessa forma, a flutuação moderada de um dos parâmetros, ou ambos, podem afetar à abundância de $X$. kroyeri na Foz do Rio Itajaí-Açú.

Coelho \& Santos (1993), analisando a proporção média anual de $X$. kroyeri nos desembarques na região de Tamandaré, PE, constataram que a população manteve o equilíbrio de 1:1, embora com abundância média das fêmeas $(54,6 \%)$ e dos machos $(45,4 \%)$. Nesse estudo, a proporção de 1:1 ficou próxima da esperada, quando considerado o total de exemplares coletados. Mensalmente, constatou-se disparidade nessa proporção com domínio significativo das fêmeas, em alguns meses, e dos machos, apenas em outubro. Em relação ao tamanho, constatouse o predomínio significativo das fêmeas entre os exemplares de até $6,0 \mathrm{~cm}$ e nos de 11,0 a $12,0 \mathrm{~cm}$, com equilíbrio nas classes de maior ocorrência.

As fêmeas dos camarões Penaeidae sempre alcançam comprimento total superior ao dos machos (Boschi, 1969). Os resultados acima mencionados corroboram esse padrão. Assim, o comprimento total máximo registrado para $X$. kroyeri, ao longo da sua área de distribuição geográfica foi de $11,5 \mathrm{~cm}$ para os machos e 14,0 cm para as fêmeas (Pérez Farfante, 1978). Os resultados obtidos nesse estudo confirmam o padrão da espécie, com $12,0 \mathrm{~cm}$ nos machos e $14,0 \mathrm{~cm}$ nas fêmeas.

De acordo com Nakagaki et al. (1995) a população de $X$. kroyeri da Enseada de Ubatuba, SP, apresentou flutuações sazonais na abundância, com as menores freqüências ocorrendo durante o verão. Neste estudo, confirma-se o padrão sazonal de ocorrência da espécie, embora as menores taxas de captura tenham sido registradas na primavera.

As informações disponíveis sobre o ciclo de vida de $X$. kroyeri indicam que não existem migrações de recrutamento ao estoque adulto, possibilitando a ocorrência de juvenis e adultos na mesma área de coleta (Vieira, 1947; Iwai, 1973). Padrão semelhante foi observado neste estudo, demonstrando que a estrutura sazonal de comprimentos da população é mantida em função dos eventos do ciclo de vida da espécie.

O conhecimento do tamanho de primeira maturação gonadal é fundamental para a administração racional dos estoques de camarões, pois fornece a informação básica para a determinação do tamanho mínimo de captura e dimensionamento das malhas das redes. Além disso, a sobreposição desse tamanho com as curvas de distribuição de comprimento permitem determinar o estrato da população em que a pesca vem atuando com maior intensidade.

Nesse estudo, os machos atingiram tamanho de primeira maturação com comprimento total de $7,4 \mathrm{~cm}$ e comprimento de carapaça de $1,39 \mathrm{~cm}$, enquanto que nas fêmeas esse tamanho foi superior, com comprimento total de $9,0 \mathrm{~cm}$ e comprimento de carapaça de $1,71 \mathrm{~cm}$. Esses tamanhos foram alcançados com idade entre 6 a 8 meses.

O tamanho de primeira maturação em X. kroyeri, ao longo de sua área de distribuição no Brasil, apresenta variações inerentes aos processos de determinação ou padrões regionais de crescimento das populações. Assim, em Tamandaré, PE, o comprimento médio da carapaça das fêmeas corresponde a 1,98cm (Coelho \& Santos, 1993). Enquanto que no Ceará esse tamanho, nas fêmeas, aumentou para 2,50cm (Mota Alves \& Rodrigues, 1977). No litoral de São Paulo, exemplares com tamanho inferior a $2,0 \mathrm{~cm}$ de comprimento da carapaça foram considerados imaturos (Vieira, 1947).

Os resultados obtidos nesse estudo apresentaram os menores tamanhos e idade em que a população de $X$. kroyeri alcança a primeira maturação, registrados no litoral brasileiro. Entretanto, a amplitude de comprimento é compatível com a literatura. 
Segundo Garcia (1988) a temperatura da água exerce papel relevante no ciclo de vida dos Penaeidae ocasionando crescimento sazonal nas áreas onde as diferenças são pronunciadas. Assim, a ampla distribuição geográfica da espécie na costa oeste do Oceano Atlântico, provavelmente seja a causadora das divergências observadas no tamanho de primeira maturação e período reprodutivo.

A relação peso/comprimento tem sido utilizada para facilitar a estimativa do peso de um exemplar, através do conhecimento do seu comprimento, sendo amplamente empregada em estudos de dinâmica populacional e avaliação de estoques. Em geral, os camarões Penaeidae apresentam uma tendência de crescimento alométrico diferenciado entre os sexos (Boschi, 1969; Melo, 1973; Zenker \& Agnes, 1977). Esse padrão, também foi observado na população de camarão sete-barbas da Foz do Rio Itajaí-Açú.

A praticidade do índice biométrico foi constatada na obtenção das curvas em crescimento da carapaça de X. kroyeri. Em geral, nos camarões Penaeidae, os machos apresentam constante de crescimento (K) mais elevada que as fêmeas em comprimentos assintóticos menores (Garcia \& Le Reste, 1981). Padrão semelhante de crescimento foi registrado nas populações de X. kroyeri da região Sudeste-Sul do Brasil, onde o comprimento médio máximo oscila entre 12,1 a $16,2 \mathrm{~cm}$ e a constante catabólica entre 0,226 a 0,6187 (Branco, et al., 1994; Nascimento \& Poli, 1986; Santos et al., 1969).

De acordo com Branco et al. (1994), as curvas de crescimento em peso de X. kroyeri sugerem que os machos crescem mais rapidamente que as fêmeas, atingindo em média, menos peso para a mesma classe de comprimento. Comportamento semelhante foi observado nesse estudo, onde os pesos máximos registrados para a espécie estiveram próximos dos pesos assintóticos estimados.
Nascimento \& Poli (1986) indicam ausência de dimorfismo sexual quanto ao comprimento total de $X$. kroyeri na Baía de Tijucas, SC. Entretanto, quando comparamos os comprimentos e pesos assintóticos com a população da Foz do Rio ItajaíAçú, torna-se evidente a existência de dimorfismo sexual e o emprego de equações distintas de crescimento para a espécie.

Segundo Iwai (1973), os camarões do gênero Penaeus apresentam um ciclo de vida relativamente curto, variando entre 16 a 50 meses. Esse padrão segue a tendência registrada para $X$. kroyeri, onde o comprimento máximo obtido na população pode ser atingido em cerca de 12 meses de idade.

\section{AGRADECIMENTOS}

À Pró-Reitoria de Pesquisa e Pós-Graduação da UNIVALI, pelo auxílio econômico e bolsa de iniciação científica ProBIC concedida a C. R. GUERRA. Ao Diretor da FACIMAR, pelas facilidades colocadas a nossa disposição. Bem como, aos estagiários do Laboratório de Oceanografia Biológica (Zoologia), acadêmicos: Willian Guimarães Vale, Rodrigo Schveitzer, pelo auxílio nas coletas e processamento das amostras.

\section{REFERÊNCIAS}

Bertalanffy, L. von. (1938), A quantitative theory of organic growth. Hum. Biol., 10(2): 181-213.

Boschi, E. E. (1969), Estudio biológico pesquero del camarón Artemesia longinaris Bate, de Mar del Plata. Boln. Biol. Mar., Mar del Plata, Argentina, 18:1-47.

Branco, J. O.; Lunardon-Branco, M. J.; De Fenis, A. (1994), Crescimento de Xiphopenaeus kroyeri (Heller, 1862) (Crustacea: Natantia: Penaeidae) da região de Matinhos, Paraná, Brasil. Arq. Biol. Tecnol., 37(1):1-8.

Coelho, P.A.; Santos, M. C. F. (1993), Época da reprodução do camarão-sete-barbas, Xiphopenaeus kroyeri (Heller, 1862) 
(Crustacea, Decapoda, Penaeidae) na região de Tamandaré, PE. Técn. Cient. CEPENE, Rio Formoso, 1(1):171-186.

D'Incao, F. (1984), Estudo sobre o crescimento de Penaeus (Farfantepenaeus) paulensis Pérez Farfante, 1967 da Lagoa dos Patos, RS, Brasil (Decapoda, Penaeidae). Atlântica, Rio Grande, 7:73-84.

Garcia, S.; Le Reste, L. (1981), Life cicles, dynamics, exploitation and management of coast penaeid shrimp stocks. FAO Fish. Tech. Pap., 203:1-215.

Garcia, S. (1988), Tropical penaeid praws. In: Fish population dynamics. Gulland, J.A. (Ed.) John Wiley \& Sons Ltd. p. 219-249.

Gunter, G. (1950), Seasonal population changes and distributions as related to salinity, of certain invertebrates of the Texas Coast, including the commercial shrimp. Publ. Inst. Mar. Sci. Univ. Tex., 1(2):1-52.

Holthuis, L. B. (1980), Shrimp and prawns of the world. An annoted catalogue of species of interest to fisheries. FAO Fish. Synop., 125(1): 1-261.

Iwai, M. (1973), Pesca exploratória e estudo biológico sobre o camarão na costa Centro/Sul do Brasil com o Navio Oceanográfico "Prof. W. Besnard" em 1969-1971. SUDELPA / IOUSP, São Paulo, $71 \mathrm{p}$.

Kinne, O. (1964), The effects of temperature and salinity on marine and brackish water animals. Oceanogr. Mar. Biol. Annu. Rev., 2: 281-339.

Matsuura, Y. (1986), Contribuição ao estudo da estrutura oceanográfica da Região Sudeste entre Cabo Frio (RJ) e Cabo de Santa Marta Grande (SC).Ciênc. Cult., 38(8):1439-1450.

Melo, J. T. C. (1973), Estudo populacional do "camarão-rosa" Penaeus brasiliensis (Latreille, 1817) e Penaeus paulensis Pérez Farfante 1967. Bol. Inst. Pesca, São Paulo, 2(2):19-65.
Mota-Alves, M. I. \& RODRIGUES, M. M. (1977), Aspectos da reprodução do camarão-setebarbas Xiphopenaeus kroyeri (Heller) (Decapoda, Macrura), na costa do Estado do Ceará. Arq. Ciênc. Mar., 17(1):29-35.

Nakagaki, J. M.; Negreiros-Fransozo, M. L.; Fransozo, A. (1995), Composição e abundância de camarões marinhos (Crustacea, Decapoda, Penaeidae) na Enseada de Ubatuba, Ubatuba (SP), Brasil. Arq. Biol. Tecnol., 38(2):583-591.

Nascimento, P. A. M.; Poli, C. R. (1986), Curva de crescimento do camarão sete-barbas, Xiphopenaeus kroyeri (Heller, 1962), na Baía de Tijucas - Santa Catarina. In: Anais do I Seminário sobre Ciências do Mar da UFSC. (1):37-41.

Neiva, G. S.; Wise, J. P. (1963), The biology and fishery of the sea bob shrimp of Santos Bay, Brazil. Proc. Gulf. Caribb. Fish. Inst., 16: 131139.

Pérez Farfante, I. (1978), Shrimps and prawns. In FISHER, W. (Ed.), FAO species identification sheets for fishery purposes.Western Central Atlantic (Fishery Area 31). Roma: FAO. V.6.

Pérez Farfante, I. (1970), Diagnostic characters of juveniles of the shrimps Penaeus aztecus aztecus, $P$. duorarum duorarum, and $P$. brasiliensis (Crustacea, Decapoda, Penaeidae). Spec. Sci. Rep. Fish., 599: 1-26.

Santos, E. P.; Neiva, G. S.; Schaeffer, Y. (1969), Dinâmica da População do camarão-setebarbas, Xiphopenaeus kroyeri (Heller) na Baía de Santos. Pesc. Pesquis., 2(2):41-55.

Santos, E. P. (1978), Dinâmica de populações aplicada à pesca e piscicultura. São. Paulo, HUCITEC / EDUSP. 129p.

Staples, D. J.; Heales, D. S. (1991), Temperature and salinity optima for growth and survival of juvenile banana praws, Penaeus merquiensis. J. Exp. Mar. Biol. Ecol., 154:251-274.

Vazzoler, A. E. A. M. (1981), Manual de métodos para estudos biológicos de populações de 
peixes: reprodução e crescimento. Brasília, CNPq. Progama Nacional de Zoologia. 106 p.

Vieira, B. B. (1947), Observações sobre a maturação de Xiphopenaeus kroyeri no litoral de São Paulo. Bol. Mus. Nacional, Rio de Janeiro, 74: 1-22.

Villela, M. J.; Costa, P. A.; Valentin, J. L. (1997), Crescimento e mortalidade de juvenis do camarão-rosa (Penaeus brasiliensis Latreille, 1817) na Lagoa de Araruama, Rio de Janeiro. Rev. Brasil. Biol., 57(3):487-499.
Walford, L. A. (1946), A new graphic method of describing the growth of animals. Biol. Bull., 90(2): 141-147.

Zenker, H. H.; Agnes, J. L. (1977), Distribuição do camarão-rosa Penaeus brasiliensis e Penaeus paulensis ao longo da costa Sudeste e Sul do Brasil. S. Doc. Tec., SUDEPE-PDP, 21:1-105.

Received: June 08, 1998; Revised: October 13, 1998; Accepted: January 07, 1999 